\title{
Sustained drug delivery of capecitabine using natural (bee wax) and synthetic polymer (PLGA)
}

\begin{abstract}
Sustained/controlled release is designed to release a drug at a predetermined rate in which a constant drug concentration maintained for a specific period with minimal side effects and drug consumption. This can be achieved via various formulations, including liposomes, solid lipid microspheres and drug-polymer conjugates. The role of polymer in drug delivery is to provide weight, consistency and volume for the right and proper administration of the drug. It provides synergistic functions, stability, drug release, targeting, enhancing its bioavailability and patient acceptability. Beeswax, a natural polymer is considered as a base biomaterial for preparation of microspheres and loading with Capecitabine, an anti-cancer drug for drug delivery. The Beeswax microspheres via coacervation and phase separation method were in the mean diameter of $3441 \mathrm{~nm}$ and SEM micrograph reveals the distribution of beeswax microsphere in Poly Lactic Glycolic Acid (PLGA) medium uniformly. The interaction between beeswax, PLGA and drug were interpreted by FTIR and confirming the drugpolymer interactions. In-vitro drug release of Beeswax microspheres confirms a linear behaviour with $26 \%$ of the drug released in the dissolution medium at $\mathrm{pH} 6.8$. The cell culture confirms the good biocompatibility of beeswax microspheres concluding the beeswax could be used to fabricate the drug delivery and targeting vehicles in cancer therapy.
\end{abstract}

Keywords: beeswax, PLGA, capecitabine, microspheres, in-vitro drug release and cell culture
Volume 2 Issue 3 - 2018

Subha V, Arulsha W, Kirubanandan S,
Renganathan S
Department of Biotechnology,Anna University, India

Correspondence: Kirubanandan S, Department of Biotechnology, Anna University, India,

Email skirubanandan80@gmail.com

Received: May 31, 2018 | Published: June 21, 2018

\section{Introduction}

Drug delivery is a platform for formulating a system for transporting a pharmaceutical compound in the body as needed to safely achieve its desired therapeutic effect with a minimal dosage. It involves site specific-targeting within the body or involving facilitating systemic pharmacokinetics with both quantity and duration of the drug at the site of delivery. It can be functionalized via a drug's chemical formulation, but it may also involve medical devices or drug-device combination products. This system provides the plasma and tissue drug levels in the body and preventing any damage to the healthy tissue via the drug toxicity. Smart polymers used in the fabrication of drug delivery systems. These polymers have the ability to act on external stimuli like light, pressure, temperature, $\mathrm{pH}$, electric field, etc., Polymers considered in designing drug delivery system should provide a considerable weight, solution consistency and volume for the proper administration of the drug and in addition, they are multi-tasking offering stability of the drug at the site of injury, drug release, targeting to the specific part of organs or tissues, enhanced bioavailability and patient acceptability. Polymers are classified based on the following groups: source (Natural, semi-synthetic, synthetic); structure of polymer (Linear, Branched-chain, Crosslinked or Network polymers); type of polymerization (Addition, condensation polymers); molecular forces (Elastomers, Fibres, Thermoplastic, Thermosetting); Chain-growth polymerization (Free radical governed); degradability (biodegradable, non-biodegradable). ${ }^{1}$ Both natural and synthetic polymers are used in drug delivery design and targeting. Natural polymers are usually large molecular weights from a natural source such as plants, micro-organisms and animals. Natural polymers are capable to tailor its properties due to low toxicity, renewability, flexibility to modification, biodegradability and low cost. Natural polymers usually made up of polysaccharides or proteins are hydrophilic, enzymatically degradable. ${ }^{2}$ Polymers like Beeswax are hydrophobic in nature which is inert and biodegradable. It has good hemocompatibility and high interaction with living cells making them compatible and suitable biomaterials for long systemic circulation and targeting. Most of the natural polymers are generally regarded as a safe zone for oral consumption and degradable via a metabolic pathway in the human body.

\section{Bio-synthesis of beeswax}

Beeswax is produced by the work bee Apis mellifera $\mathrm{L}$. Around the world, beeswax was produced from A.cerana and A. florae as well as other honeybee species. Formation and secretion of wax take place at gland complexes consisting of three cell types: epithelial cells, oenocytes and adipocytes, which act synergistically to secrete wax. It is a constant process and starts in a week old honey bee workers, goes a peak after 2 weeks of age and thereafter decreases. ${ }^{3}$

\section{Composition of beeswax}

Beeswax is a unique complex mixture of both saturated and unsaturated linear and complex monoesters, hydrocarbons, free fatty acids, free fatty alcohols and other minor exogenous substances. It is reported that a normal bee wax consists of a total content of 27 to $40 \%$ monoesters, 9 to $23 \%$ hydroxy-monoesters, 7 to $16 \%$ diesters, $3.9 \%$ hydroxy-diesters, 11 to $28 \%$ hydrocarbons, 1 to $18 \%$ saturated, unbranched free fatty acids, 4 to $8 \%$ of other substances and less than $0.3 \%$ free fatty alcohols from different bees species. ${ }^{4}$ 


\section{Properties of beeswax}

Melting range-between $62^{\circ} \mathrm{C}$ and $65^{\circ} \mathrm{C}$ Specific gravity $\left(\mathrm{D}_{2} 0\right)$ about 0.96 , Solubility-Insoluble in water, Sparingly soluble in alcohol. Very soluble in chloroform and ether, Purity Acid value-not less than 17 and not more than 24 (mg KOH/g beeswax) Saponification value-87-104 ( $\mathrm{mg} \mathrm{KOH} / \mathrm{g}$ beeswax) Peroxide value $\left(\mathrm{mM} \mathrm{H}_{2} \mathrm{O}_{2} / 1000 \mathrm{~g}\right.$ beeswax): not more than 5 Glycerol and other polyols: not more than $0.5 \%$ (as glycerol) Ceresin, paraffin and certain other waxes: absent The synthetic polymer has more advantages and their physicochemical properties tailored for designing drug delivery system. Especially, synthetic water-soluble polymers modify its physical properties of aqueous systems in the form of gelation, thickening or emulsification/ stabilization when it dissolves in water or aqueous medium. These polymers usually have repeating units or blocks of units (e.g. PEG); the polymer chains contain hydrophilic groups that are substituents or are incorporated into the backbone. The hydrophilic groups may be non-ionic, anionic, cationic or amphoteric. ${ }^{5}$

\section{Poly (lactic-co-glycolic acid)}

PLGA or Poly (lactic-co-glycolic acid) is a copolymer, and an approved polymer by Food and Drug Administration (FDA) for developing therapeutic and medical devices due to its outstanding biodegradability and biocompatibility with biological systems. It is made by a process of ring-opening copolymerization of two different monomers, the cyclic dimers (1,4-dioxane-2,5-diones) of glycolic acid and lactic acid. PLGA is a successful biodegradable polymer. It undergoes hydrolysis in the body to produce the original monomers, lactic acid and glycolic acid, a metabolite which is excreted easily in the body via various metabolic pathways. These monomers have very minimal systemic toxicity for constructing drug delivery devices or biomaterial scaffold for tissue engineering applications Figure 1.

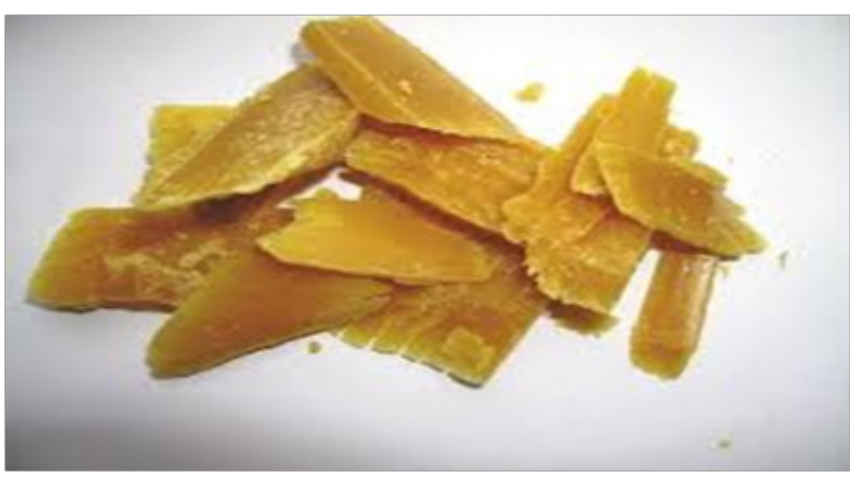

Figure I crude beeswax.

\section{Physical properties}

Composition: PLGA copolymer, 50:50LAGA or 75:25LAGA. Structure: Amorphous Density: 50:50PLGA-1.34g/ $\mathrm{cm}^{3}, 75: 25 \mathrm{PLGA}-$ $1.30 \mathrm{~g} / \mathrm{cm}^{3}$ Glass Transition Temperature: 50:50 PLGA-44-55 ${ }^{\circ} \mathrm{C}$, 75:25PLGA-50-55 ${ }^{\circ} \mathrm{C}$. Molecular Weight: 50:50PLGA- 150,000, 75:25PLGA- 90,000. Degradation pH: highly acidic (upto 2.5 ).

\section{Model drug}

Capecitabine is a prodrug of 5-fluorouracil which is inactive, and this prodrug converted into an active drug via enzymatic conversion occurring only in tumour tissue. ${ }^{6}$ It is an oral prodrug of 5-FU which passes as a native formulation through the gut wall and then converted into 5'dFCR following changed to 5'-deoxy-5-fluorouridine ( 5 ' dFUR) in the liver via enzymatic reaction with carboxylesterase and cytidine deaminase respectively. 5'dFUR is finally then converted to 5-FU via thymidine phosphorylase or uridine phosphorylase. ${ }^{6,7}$ The main mechanism of 5-FU activation is conversion to fluorodeoxyuridine monophosphate (FdUMP) which inhibits the enzyme thymidylate synthase (TYMS), a component of the folatehomocysteine cycle and purine and pyrimidine synthesis Table 1. This paper deals the synthesis of beeswax microspheres by using melt dispersion method and to evaluate the drug loading capacity of the microspheres by using a model anti-cancer drug capecitabine and its drug release studies and to coat the PLGA polymer incorporated with the model drug, on the outer surface of the beeswax microspheres and its characterization studies Figure 2.

Table I pharmacokinetic data

\begin{tabular}{ll}
\hline Bioavailability & Extensive \\
\hline Protein binding & $<60 \%$ \\
Metabolism & $\begin{array}{l}\text { Hepatic, to 5'- DFCR, 5'- DFUR (inactive); } \\
\text { neoplastic tissue, 5'- DFUR to active fluorouracil }\end{array}$ \\
Biological half- life & $38-45$ minutes \\
Excretion & Renal $(95.5 \%)$, faecal $(2.6 \%)$ \\
\hline
\end{tabular}

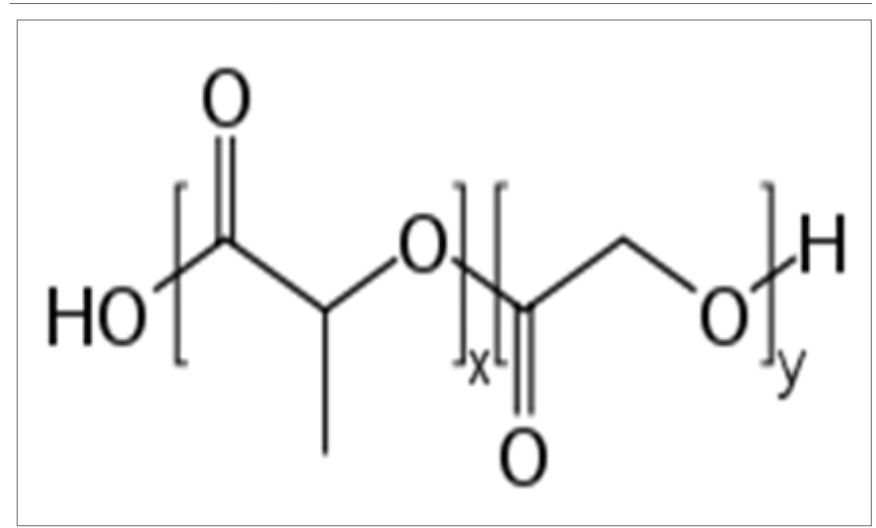

Figure 2 Structure of PLGA. where, $X=$ no of units of lactic acid and $Y=$ no of units of glycolic acid.

\section{Materials and methods}

\section{Collection of beeswax and PLGA}

Crude beeswax (yellow) was collected from Dindigul, Tamil nadu, India. It was further melted and filtered to remove the impurities. PLGA (50:50) was purchased from Sigma Aldrich.

\section{Synthesis of beeswax microsphere}

1.5grams of Beeswax was melted separately in a hot air oven at a temperature of $60 \pm 1{ }^{\circ} \mathrm{C}$. Model Drug $(0.13 \mathrm{gm})$ was added to the melted condition of wax and stirred and dispersed to obtain a homogeneous melt. This mixture was poured into $33 \mathrm{ml}$ of Ammonia buffer solution for minimizing the solubility of the drug at $\mathrm{pH}$ 10.9. While adding the ammonia buffer solution, the wax is heated and melted just higher than $65 \pm 1^{\circ} \mathrm{C}$. Tween $80(1.8 \% \mathrm{w} / \mathrm{w})$ was also added to the mixture. This mixture was well stirred at 900RPM using a stirrer (RQ-127A) fitted with a 4-blade impeller of $53 \mathrm{~mm}$ diameter. During mixing 
process, spherical particles containing drug and wax are produced due to the dispersion of molten wax in the aqueous medium. The mixture was stirred continuously at $900 \mathrm{rpm}$ at a higher temperature $\left(65^{\circ} \mathrm{C}\right)$ of the melting point of wax for $4 \mathrm{~min}$. The temperature of the mixture in the beakers was cooled rapidly to $10^{\circ} \mathrm{C}$ by the addition of cold water Figure 3. The resultant solid spheres were collected by filtration were extensively washed with water to remove drug adsorbed on the surface and surfactant residues. Air drying was carried out at room temperature for $48 \mathrm{~h}$ to get free-flowing solid microspheres.

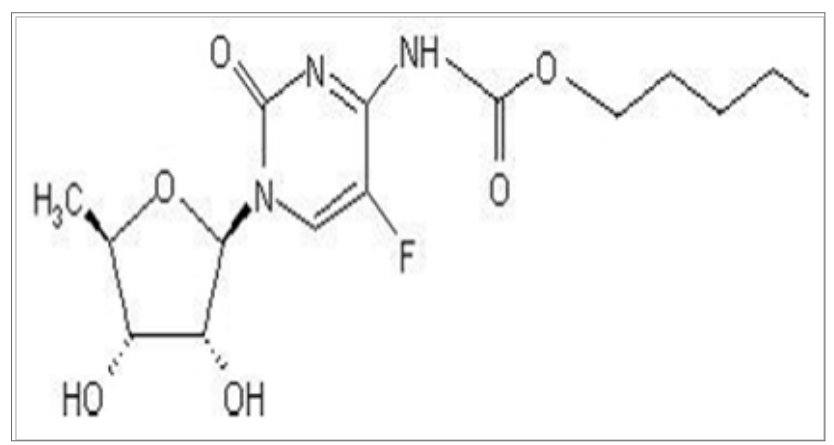

Figure 3 Structure of capecitabine.

\section{Preparation of PLGA-drug solution by phase separation method}

Coacervation is a process preparing a microsized biodegradable polymer via liquid-liquid phase separation method. In this method, it yields two liquid phases via phase separation namely the polymer containing coacervate phase and the supernatant phase depleted in the polymer. The drug which is dispersed/dissolved in the polymer solution is coated by the coacervate. ${ }^{8}$ In this study, PLGA Polymer mixed with a solvent $(800 \mu \mathrm{l})$ in appropriate ratios to form a solution. The drug capecitabine $(10 \mathrm{mg})$ was dissolved in water $(1 \mathrm{ml})$ and dispersed in the polymer solution (water-in-oil emulsion). The gradual addition of organic medium to the polymer-drug-solvent solution phase extracts the polymer solvent resulting in phase separation of the polymer by forming a soft coacervate of drug-containing droplets. The size of the droplets was controlled by varying stirring rate and temperature of the system. After vortex at 1000rpm and ultrasonication for 7000rpm, the resultant solution could air dry at room temperature for $24 \mathrm{hrs}$ for solvent evaporation. For next $24 \mathrm{hrs}$, the solution kept in refrigeration to keep that in a liquid phase. Already synthesized drug loaded beeswax microspheres were mixed with this solution which made dispersion. In-room temperature PLGA obtained gel-like consistency Figure 4. It has the ability of up taking water and gets swollen. Thus, it will act as a hydrogel at the site of injury and swells in a physiological environment.

\section{Characterization studies}

The synthesized micro-sized polymer was characterized by FTIR (Model Name FT/IR-6300 type), UV visible spectrophotometer (UV 1800-Shimadzu), SEM (Zeiss) and PSA.

\section{In vitro drug release}

In vitro release of Capecitabine from microspheres was studied using a USP dissolution apparatus (Pharma test). Phosphate buffer solutions of $\mathrm{pH} 6.8$ were used as dissolution medium, their $\mathrm{pH}$ was adjusted by adding $\mathrm{HCl}$ or $\mathrm{NaOH}$ solutions Table 2. $100 \mathrm{mg}$ of microspheres were added in cellulose dialysis tube containing $4 \mathrm{ml}$ of dissolution medium and tied to the paddle. The paddle was rotated at $100 \mathrm{rpm}$ in $500 \mathrm{ml}$ of phosphate buffer solution of $\mathrm{pH} 6.8$ at $37^{\circ} \mathrm{C}$. A $2 \mathrm{ml}$ sample was withdrawn at predetermined time intervals i.e. 0 , $1,2,3,4,5,6,8$ and $24 \mathrm{~h}$ and an equal volume of fresh dissolution medium, which was prewarmed at $37^{\circ} \mathrm{C}$, was replaced. Collected samples were suitably diluted and then analysed for Capecitabine contents by measuring the absorbance at $283 \mathrm{~nm}$ using an ultraviolet spectrophotometer (1800-Shimadzu UV-spectrophotometer). The concentration of Capecitabine in test samples was calculated using calibration curve.

Table 2 show different stretches of bonds shown at different peaks

\begin{tabular}{ll}
\hline Peaks $\left(\mathbf{C m}^{-1}\right)$ & Functional groups \\
\hline $2918-2850$ & C-H stretch \\
1736 & C=O stretch \\
1173 & Easters \\
$1750-1760$ & Carbonyl Group \\
$1200-1000$ & C-OH \\
$1160-1083$ & Ether group \\
1408 & Aromatic stretch \\
\hline
\end{tabular}

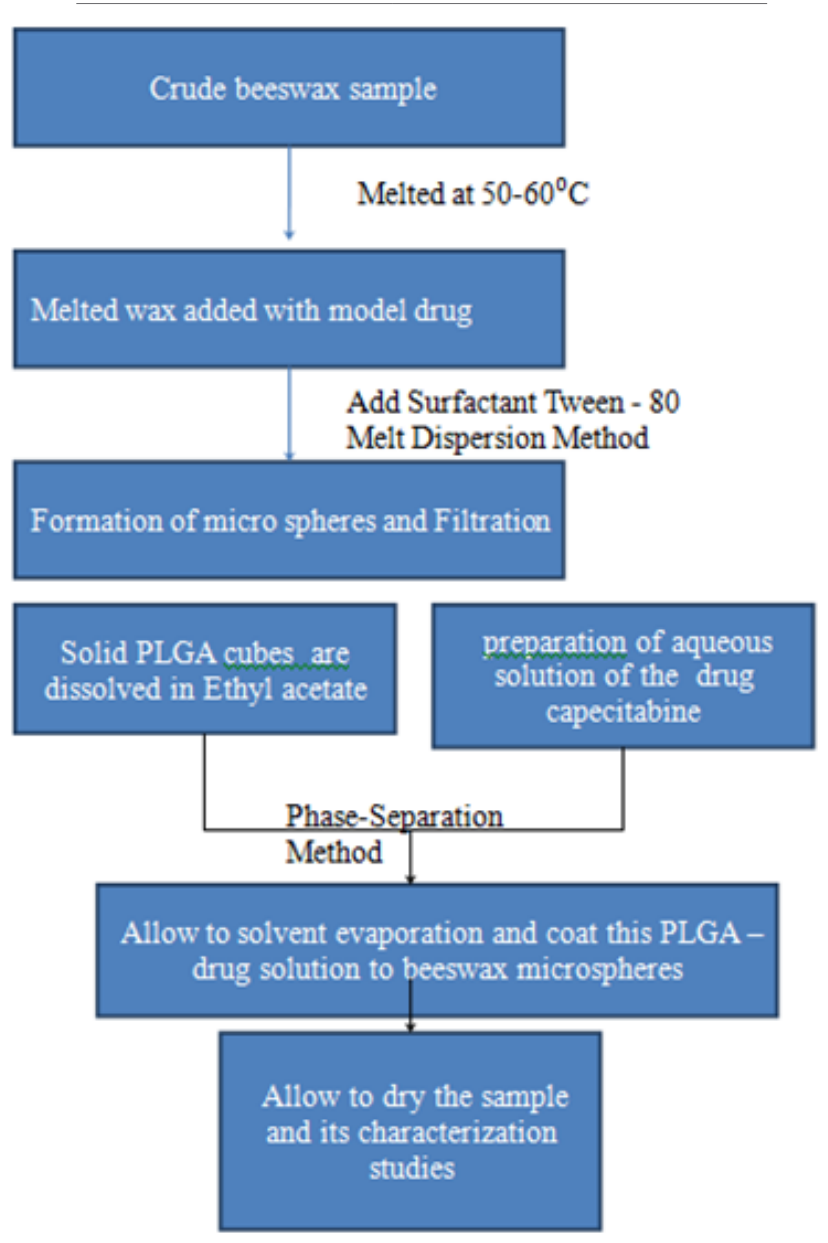

Figure 4 Preparation of drug delivery vehicles. 


\section{Cell passaging}

In this study in vitro biocompatibility of the beeswax, microspheres were carried out on Human Umbilical Vein Endothelial Cells (HUVEC) EA. hy 926. Confluent grown cell culture was bought as a gift sample from cell culture lab, Anna University MIT campus, Chennai. Confluent grown cells were resuspended in $1 \mathrm{ml}$ of $1 \mathrm{X}$ PBS and mixed gently. This mixture was added with $1 \mathrm{ml}$ of Trypsin Phosphate Versene Glucose 1X. Trypsin was pipetting out and the flask was kept in an incubator for 2 minutes and then checked for detachment of cells. After that $1 \mathrm{ml}$ of cell culture media was added and mixed well.

\section{Cell counting}

Several cells present in cell culture flask was calculated using Neubauer chamber. $10 \mu 1$ of cells and $10 \mu 1$ Tryphan blue dye was added and mixed well. $10 \mu \mathrm{l}$ of this sample mixture was taken and poured under the coverslips and chamber. This was viewed under microscope and cells were counted using the formula given below. No of cells in $1 \mathrm{ml}=$ Total no of cells in 16 small squares $* 2$ (dilution factor $)^{*} 10^{4}$

\section{Result and discussion}

The beeswax microspheres were synthesized. The size of the spheres was trying to be reduced by increasing the stirring rpm and the time of stirring was extended up to 30 minutes.

\section{Particle size analyzer}

Particle size analysis was performed by dynamic light scattering (DLS) with a Malvern Zetasizer 3000HSA (Malvern Instruments, UK). Figure 5A \& 5B shows particle size distribution of beeswax microspheres. DLS yields the mean diameter and the polydispersity index (PI) which is a measure of the width of the size distribution. The mean diameter and PI values were obtained at an angle of $90^{\circ}$ in $10 \mathrm{~mm}$ diameter cells at $25^{\circ} \mathrm{C}$. Prior to the measurements, all samples were diluted with double distilled water to produce a suitable scattering intensity. The synthesized microspheres were in the mean diameter of $3441 \mathrm{~nm}$ or $34 \mu \mathrm{m} .{ }^{9,10}$

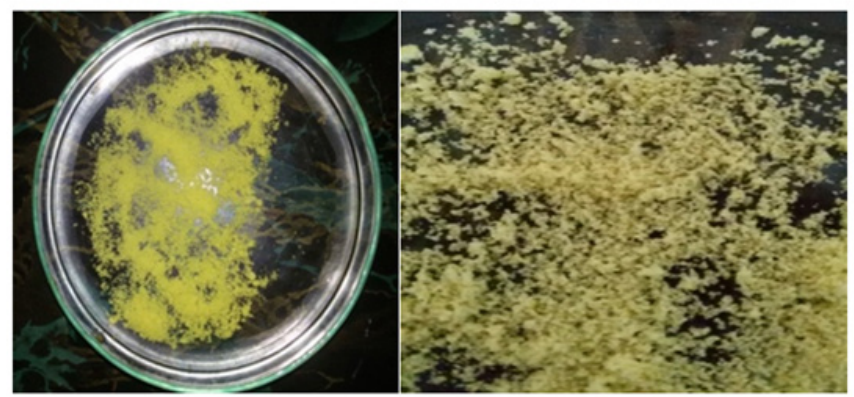

Figure 5 (A) beeswax microspheres, (B) beeswax microspheres loaded with the model drug (capecitabine).

\section{Scanning electron microscope}

Surface morphology of the microspheres was observed on the scanning electron microscope (Zeiss-Model S3). From the Figure 6, the distribution of beeswax microsphere in PLGA was observed uniformly. The spherical shape of beeswax microspheres were easily visualised. At the elevated temperature of above $35^{\circ} \mathrm{C}$, the PLGA with the appearance of semi-solid gel phase can be easily observed with beeswax microspheres.

\section{Fourier transform infra-red spectroscopy}

Drug-polymer interactions were studied by FTIR spectroscopy (Model Name FT/IR-6300 type). The spectra were recorded for the pure drug, drug-loaded microspheres and PLGA coated microspheres using FTIR. Samples were prepared in $\mathrm{KBr}$ disks $(2 \mathrm{mg}$ sample in $200 \mathrm{mg} \mathrm{KBr}$ ). The scanning range was $400-4000 \mathrm{~cm}^{-1}$ and the resolution was $2 \mathrm{~cm}^{-1}$. The results of FTIR analysis of this study show different stretches of bonds shown at different peaks. Figure 7 shows peaks near $2918-2850 \mathrm{~cm}^{-1}$ which corresponds to $\mathrm{C}-\mathrm{H}$ stretch, peaks near $1736 \mathrm{~cm}^{-1}$ corresponds to $\mathrm{C}=\mathrm{O}$ stretch, peaks near $1173 \mathrm{~cm}^{-1}$ corresponds to a functional group of esters. In Figure 8, 1750-1760 $\mathrm{cm}^{-1}$ corresponds to a carbonyl group. $1200-1000 \mathrm{~cm}^{-1}$ region corresponds to $\mathrm{C}-\mathrm{OH}$ side groups and peaks near $1421 \mathrm{~cm}^{-1}$ corresponds to $\mathrm{C}=\mathrm{C}$ stretch, peaks near $1160-1083 \mathrm{~cm}^{-1}$ corresponds to ether group, peaks near $1408 \mathrm{~cm}^{-1}$ corresponds to aromatic stretch. Thus, we could conclude that the missing functional groups in Figure 9 may participate in the bonding of beeswax and PLGA polymers. From the release studies, it was observed that there was an initial burst of a drug in the solution due to the Swelling of PLGA. In the first $2 \mathrm{~h}$, at $6.8 \mathrm{pH}$, $6 \%$ of the drug release was observed. But after 2 hours the release of a drug was in a slow rate. The microspheres showed a sustained release pattern at $\mathrm{pH} 6.8$ (Figure 10). In 24hours the drug release was in a linear manner and approximately $26 \%$ of the drug was released in the dissolution medium of $\mathrm{pH} 6.8 .^{11-15}$

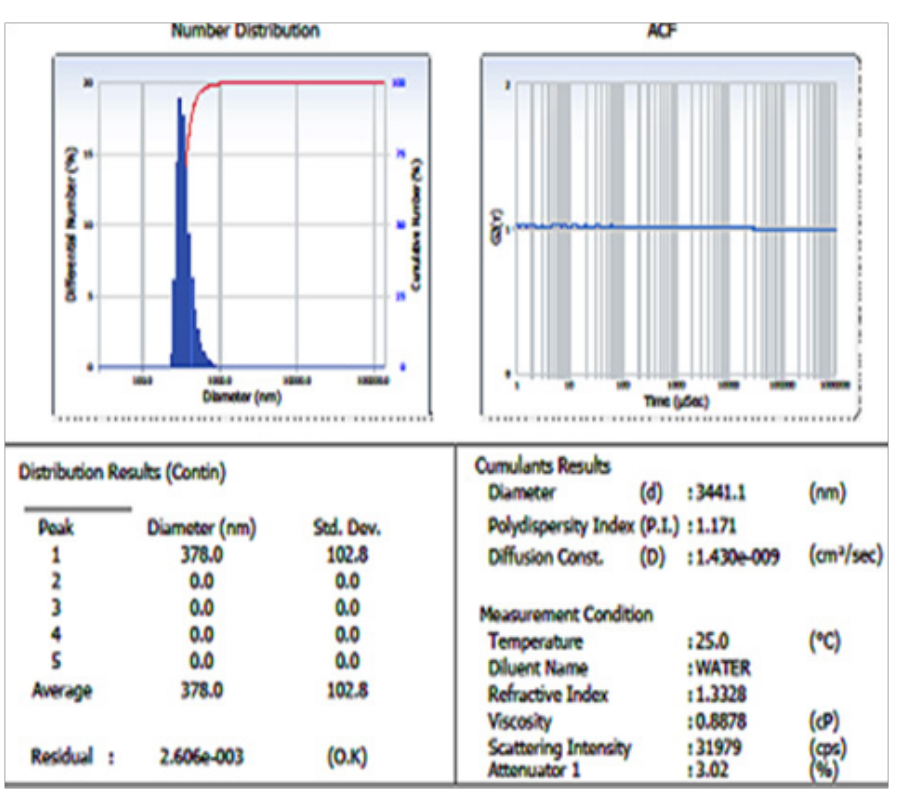

Figure 6 Particle size distribution of beeswax microspheres.

\section{Biocompatibility assay for beeswax}

The cell line Human Umbilical Vein Endothelial Cells (HUVEC) EA.hy926 were seeded in a 24 well plate as a triplicate at 3 various concentrations from lower to higher. it was further incubated for $24 \mathrm{hrs}$ in $5 \% \mathrm{CO}_{2}$ incubator. The results showed that even in higher concentration, the cells were alive (Figure 11). 


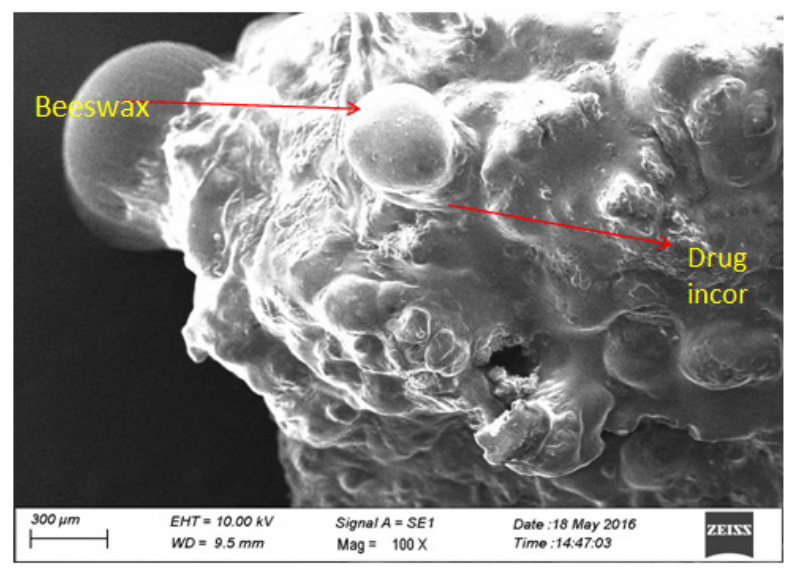

Figure 7 SEM micrograph of beeswax microspheres in PLGA.

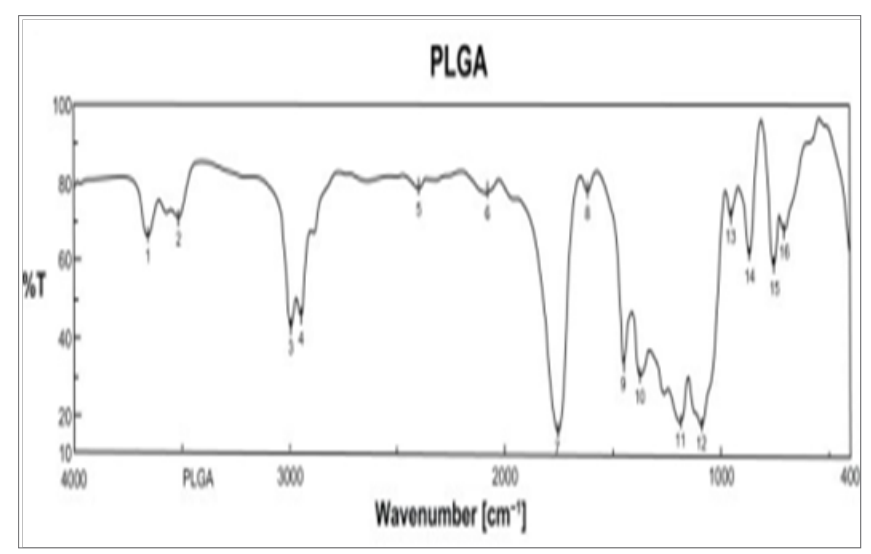

Figure 9 FTIR spectrum of PLGA/WAX microspheres.

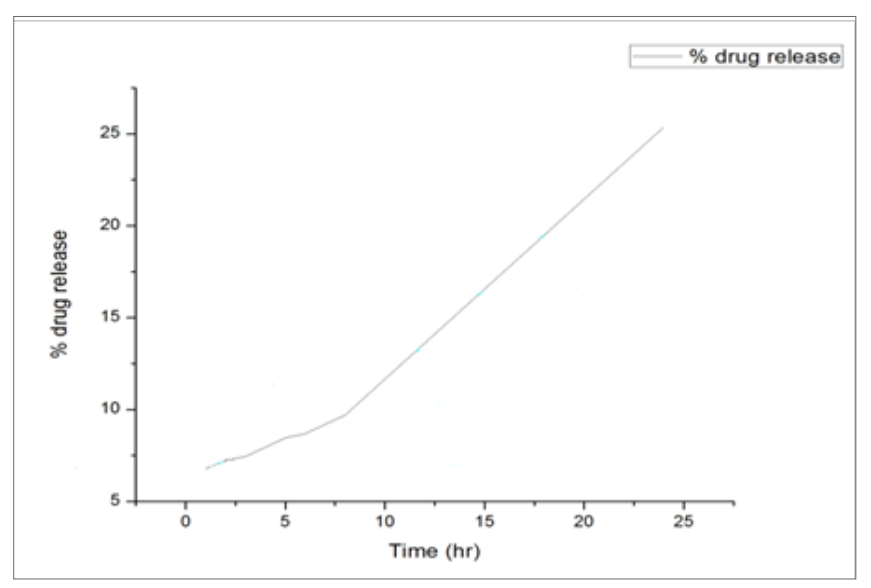

Figure II In-vitro release of drug from the microspheres.

\section{Discussion}

Recently, natural polymers are played a major role in the development of drug delivery system and other biomedical devices. Bee wax is one of the most abundant natural polymers under the

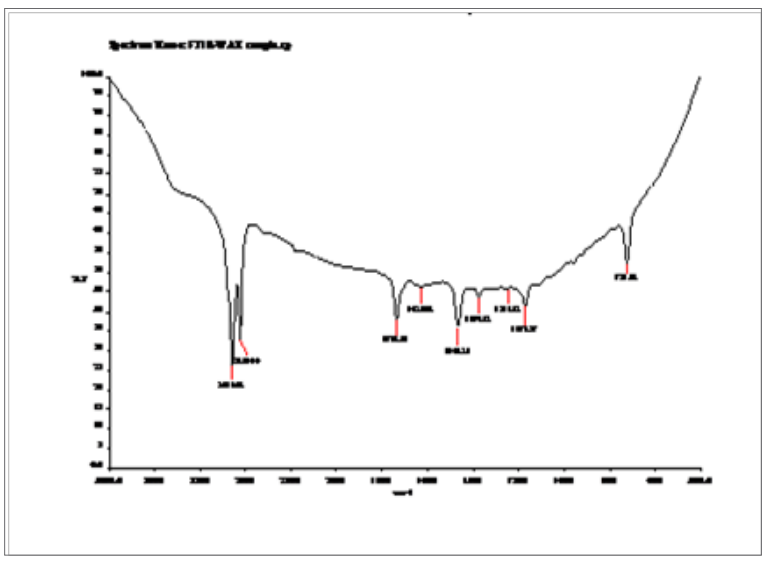

Figure 8 FTIR spectrum of pure PLGA sample.

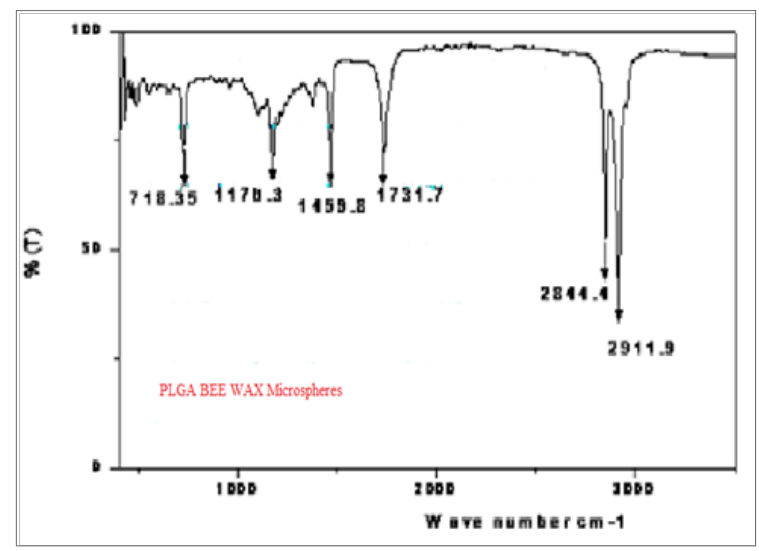

Figure I0 FTIR spectrum of PLGA/WAX microspheres.

category of wax and prepared from honeycomb as a natural source and this wax itself has potential pharmacological activities such as lowering cholesterol and pain-relieving activity. This material used as an anti-inflammatory agent for swelling, ulcers and diarrhoea. Apart from the medicinal application, it is used in food processing as a stiffening agent and a thickener and emulsifier. Additionally, it is a fragrance material in soap and perfume processing. It is declared that bee wax is a safe material for the medical and pharmaceutical application. ${ }^{16,17}$ Bee wax chemically composed of fatty acids esters and numerous long chain alcohols and edible in nature and no toxicity like plant waxes. The important components of bee wax are monoesters, hydrocarbons, diesters and free fatty acids. The physical properties of bee wax are exceptional and solid at cold temperature and tailoring its melting properties with temperature and it can be used to fabricate/formulate drug delivery vehicles such microspheres/ nanoparticles for sustained and controlled drug delivery system. Recently ${ }^{18}$ bee wax microspheres can be used as a potential drug delivery of nateglinide, an antidiabetic drug and other drugs. In this work, bee wax microspheres as drug delivery vehicles prepared via the chemical method and the size of the microspheres can be controlled by numerous process parameters such as agitation speed, wax/solvent ratio, agitation time and temperature. ${ }^{19}$ The coating of Bee wax microspheres with PLGA could increase its biocompatible 
and drug loading performance for the controlled delivery system. In the present investigation, the size of the nanoparticle was obtained as $3441 \mathrm{~nm}$ when compared with the values obtained as $4000 \mathrm{~nm}$. From this result, it was observed that the size nanoparticle was found to be comparable with the other research work established Figure 12. The SEM micrograph reveals the distribution of bee wax microspheres in the PLGA medium uniformly. The chemical processing of bee wax yields the formation of spherical micro particles having good surface area and good contact with the biological medium. FTIR confirms no chemical interaction with drug and biopolymers bee wax and PLGA. The methodology of drug release in the waxy material is controlled by erosion/diffusion of drugs. In this study ${ }^{20,21}$ drug release from the waxy formulations exhibited zero order kinetics. Due to the erosion of waxy material and diffusion of drug through the beeswax, a constant release rate was observed. From cell culture studies, it was concluded that the beeswax microspheres were biocompatible for the construction of controlled drug delivery system. ${ }^{22}$
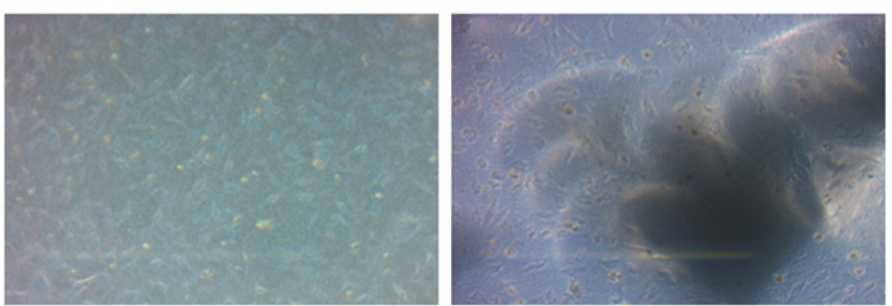

Figure 12 Cell cultures studies on beeswax microspheres like Control cell line, Cell line with beeswax.

\section{Conclusion}

The beeswax microspheres loaded with the drug were formed using melt dispersion technique. These BW microspheres construct the inner core of the drug delivery system. These BW microspheres were dispersed in the drug incorporated PLGA. This formulation played as a sustained drug delivery system for anti-cancer drugs such as capecitabine which was only suitable for oral drug administration. From SEM analysis it concludes that the beeswax microspheres were dispersed in the PLGA and formed a gel-like substance at room temperature. By focusing the in-vitro drug release study, it was accounted that the release was in a slow rate and approximately $25 \%$ of the drug from the formulation was released in 24hours, concluding the Bee Wax microspheres can be a potential drug delivery vehicles and drug targeting in cancer therapy.

\section{Acknowledgments}

None.

\section{Conflict of inertest}

Author declares that there is no conflict of interest.

\section{References}

1. Basarkar GE, Shirsath GN, Patil SB. Development of microspheres containing Diclofenac diethylamine as sustained release Topical formulation. Bulletin of Pharmaceutical Research. 2013;3(1):14-22.

2. Buchwald R, Michael D, Greenberg AR. The thermal properties of beeswaxes: unexpected findings. The Journal of Experimental Biology. 2008;211(1):121-127.
3. Budman DR. Invest New Drugs. Springer. 2000;18(4):355-363.

4. Claudio T, Hans P, Bruno A, et al. Physico-chemical parameters governing protein microencapsulation into biodegradable polyesters by coacervation. International Journal of Pharmaceutics. 1997;147(2):173-186.

5. Cortesi R, Espositoa E, Lucab G, et al. Production of lipospheres as carriers for bioactive compounds. Biomaterials. 2002;23(11):2283-2294.

6. Crow BB, Borneman AF, Hawkins DL, et al. Evaluation of in Vitro Drug Release, pH Change, and Molecular Weight Degradation of (Poly(Llactic acid) and Poly(D, L-lactide-co-glycolide) Fibers. Tissue Eng. 2005;11(8):1077-1084.

7. Edelman R, Robert G, Genevieve Losonsky, et al. Immunization of rabbits with enterotoxigenic E. coli colonization factor antigen (CFA/I) encapsulated in biodegradable microspheres of poly (lactide-coglycolide). Vaccine. 1993;11(2):155-158

8. Filippo F, Giovanni C, Barbara T, et al. Beeswax: A mini review of its antimicrobial activity and its application in medicine. Asian Pacific Journal of Tropical Medicine. 2016;9(9):839-843.

9. Gowda DV, Gupta VK, Khan MS, et al. Encapsulation of Clozapine into Beeswax Microspheres: Preparation, Characterization and Release Kinetics. International Journal of Pharm Tech Research. 2011;3(4):2199-2207.

10. Grodzinskia JJ. Polymeric gels and hydrogels for biomedical and pharmaceutical applications. Wiley Inter Science; 2009.

11. Jane L. Yen-Revollo, Richard $M$, et al. Can Inhibiting Dihydropyrimidine Dehydrogenase Limit Hand-Foot Syndrome Caused by Fluoropyrimidines? Clin Cancer Res. 2008;14(1):8-13.

12. Kamble R, Maheshwari M, Paradkar A, et al. Melt Solidification Technique: Incorporation of Higher Wax Content in Ibuprofen Beads. AAPS Pharm Sci Tech. 2004;5(4):75-83.

13. Kumara A, Srivastavaa A, Galaevb IY, Smart polymers: Physical forms and bioengineering applications. Program Polymer Science. 2007;32:1205-1237.

14. Ranjha M, Nazar Khan, Hafeezullah Naseem, et al. Encapsulation and characterization of controlled release flurbiprofen loaded microspheres using beeswax as an encapsulating agent. Journal of materials science. 2010;21(5):1621-1630

15. Makadia HK, Siegel SJ. Poly Lactic-co-Glycolic Acid (PLGA) as Biodegradable Controlled Drug Delivery Carrier. Polymers. 2011;3(3):1377-1397.

16. Ngwuluka NC, Ochekpe NA, Aruoma OI. Naturapolyceutics: The Science of Utilizing Natural Polymers for Drug Delivery. Polymers. 2014;6(5):1312-1332.

17. Cassier P, Lensky Y. Ultrastructure of the wax gland complex and secretion of beeswax in the worker honey bee Apis mellifera L. Apidologie. 1995;26(1):17-26.

18. Peppas NA. HYDROGEL. Biomaterials Science; 2003.

19. Praveen B, Lata S, Tejraj M, et al. Novel blend microspheres of cellulose triacetate and bee wax for the controlled release of nateglinide. Journal of Industrial and Engineering Chemistry. 2014;20(2):397-404.

20. Aichholz R, Lorbeer E. Investigation of combwax of honeybees with high-temperature gas chromatography and high-temperature gas chromatography-chemical ionization mass spectrometry: I. Hightemperature gas chromatography. Journal of Chromatography. 1999;855(2):601-615. 
21. Silva ATCR, Cardoso BCO, Silva MESR, et al. Synthesis, Characterization, and Study of PLGA Copolymer in Vitro Degradation. Journal of Biomaterials and Nano biotechnology. 2015;6(1):8-19.
22. Wang S, Wang H, Liu Z,et al. Smart $\mathrm{pH}$-and Reduction-Dualresponsive Folate-PEG coated Polymeric Lipid Vesicles for Tumortriggered Targeting Drug Delivery. The Royal Society of Chemistry. 2014;6(13):7635-7642. 